\title{
Graphene-Based Terahertz Closed-Stopband Tunable Composite Right/Left-Handed Leaky-Wave Antennas
}

\author{
Muzhi Gao ( $\nabla$ gaomuzhi@whu.edu.cn ) \\ Shandong University https://orcid.org/0000-0002-5748-6131 \\ Kang Li \\ Shandong University
}

Fanmin Kong

Shandong University

Gaoyang Zhu

Shandong University of Science and Technology

Huawei Zhuang

Shandong Jianzhu University

\section{Research Article}

Keywords: Graphene, Tunable, Terahertz (THz), Supercell, Narrow-band, Composite right/left-handed (CRLH), Leaky-wave antennas (LWAs)

Posted Date: February 15th, 2021

DOl: https://doi.org/10.21203/rs.3.rs-196300/v1

License: (c) (1) This work is licensed under a Creative Commons Attribution 4.0 International License. Read Full License

Version of Record: A version of this preprint was published at Optical and Quantum Electronics on August 7th, 2021. See the published version at https://doi.org/10.1007/s11082-021-03121-5. 


\title{
Graphene-Based Terahertz Closed-Stopband Tunable Composite Right/Left-Handed Leaky-Wave Antennas
}

\author{
Muzhi Gao ${ }^{1}$, Kang Li ${ }^{1}$, Fanmin Kong ${ }^{1}$, Gaoyang $\mathrm{Zhu}^{2}$, Huawei Zhuang ${ }^{3}$
}

\begin{abstract}
A simple scheme for the realization of the terahertz (THz) fundamental-mode tunable closed-stopband composite right/left-handed leaky-wave antennas (CRLH LWAs) is presented. The proposed CRLH LWAs are reconstructed by graphene-based coplanar waveguide $(\mathrm{CPW})$ transmission line supercells. Their shunt inductance achieved by narrow graphene strips of two unit cell structures are halfed. The CRLH LWAs are designed and confirmed by numerical simulations. They also exhibit frequency-scanning behaviors at $\mathrm{THz}$ with narrower bandwidth than that of the conventional graphene-based fundamental-mode CPW unit cell CRLH LWAs at THz. Besides, the LWAs also exhibit strong tunable characteristics and achieve the closed-stopband condition more effectively. Therefore, the proposed supercell CRLH LWAs could further improve the performance of the beam-steering antennas at $\mathrm{THz}$.
\end{abstract}

Keywords Graphene · Tunable · Terahertz $(\mathrm{THz}) \cdot$ Supercell $\cdot$ Narrow-band · Composite right/left-handed $(\mathrm{CRLH}) \cdot$ Leaky-wave antennas (LWAs)

\section{INTRODUCTION}

With the rapid development of the transmission rates of wireless communication, radar and satellite systems, terahertz (THz) antenna technology is a hot research area [1]. Graphene metamaterial attracts lots of attention for its potential on the

Fanmin Kong

kongfm@sdu.edu.cn

\footnotetext{
${ }^{1}$ School of Information Science and Engineering, Shandong University, Jinan 250000, China.
}

2 School of Electronic and Infromation Engineering, Shandong University of Science and Technology, Qingdao 266000, China.

3 School of Infromation and Electrical Engineering, Shandong Jianzhu University, Jinan 250101, China. development of antennas at $\mathrm{THz}$, especially in the mid-infrared region due to its unique electronic properties, strong tunable characteristics, and high electron mobility [2].

There are already lots of literature on the realization of composite right/left-handed leaky-wave antennas (CRLH LWAs) at THz. Philip has proposed the cavity antenna model for the CRLH LWAs in the THz band by the TM01 lateral mode of the reconstructed metal-metal waveguide [3]. Besides, TM01 lateral mode has also been proposed to be employed in other structures for the achievement of CRLH LWAs at THz [4], [5]. Some of these theories has been proved by experiments then [5], [6]. Besides, graphene has also been supposed have the potential for the development of the CRLH LWAs in the THz band. Derrick argues that CRLH LWAs can be realized through the first higher-order lateral mode propagated along the graphene ribbon with periodic gaps [7].

In addition, graphene-based coplanar waveguide (CPW) technology could also be applied for the development of the conventional CRLH LWAs at THz for its simple structure. In many application cases, the CRLH LWAs should satisfy a closed-stopband demand. However, the conventional graphene-based CPW unit cell CRLH LWAs cannot completely close the stopband. Therefore, the implement of the graphene-based CPW supercell technology is supposed to realize the CRLH LWAs at THz with closed-stopbands [8].

In this paper, the graphene-based CPW supercells is reconstructed to achieve the narrow-band CRLH leaky-wave structures as Fig. 1(a) shows. Their equivalent circuit model is given in Fig. 1(b). Their narrow graphene strips for realizing the shunt inductance components $L_{L}$ are halfed of each two conventional graphene-based CRLH unit cells. CST verified the proposed leaky-wave structures. Their dispersion relation is extracted from the designed leaky-wave supercell structures and its leaky-mode regions are acquired. The simulation results indicate that the scanning angle of their main radiation beams also steers obviously with the frequencies scanning from the backward-to-forward quadrant at THz. Besides, the proposed supercell CRLH LWAs achieve the closed-stopband condition more effectively than the conventional graphene-based unit cell CRLH LWAs. Furthermore, the strong tunable characteristics of the proposed supercell LWAs are discovered at THz as well. 


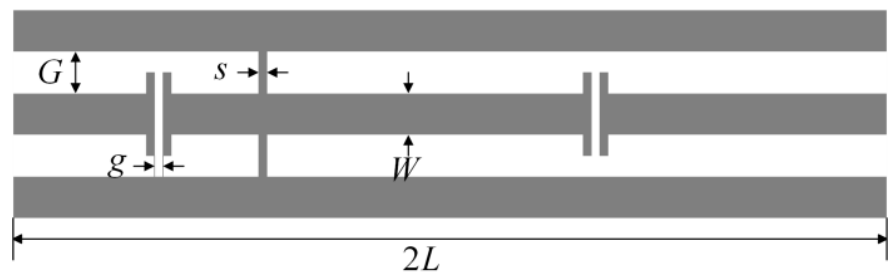

(a)

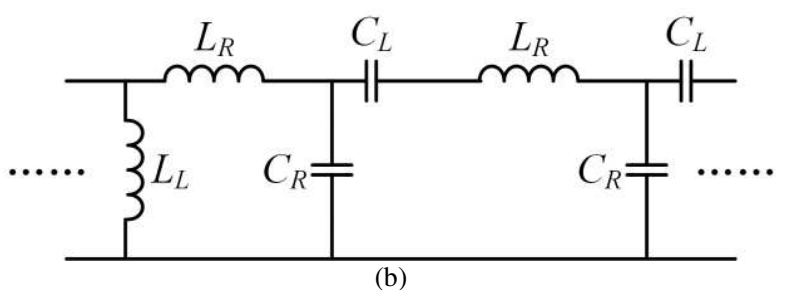

Fig. 1. The proposed supercell CRLH leaky-wave structures. (a) Layout. (b) Equivalent circuit model.

Hence, the proposed supercell LWAs would futher improve the performance of beam-steering antennas at $\mathrm{THz}$.

\section{Model Analysis}

\section{A. Surface Impedance Of Graphene}

The proposed supercell CRLH LWAs are developed by graphene metamaterial. In the low $\mathrm{THz}$ band $(<10 \mathrm{THz})$, the surface conductivity of graphene is mainly dominated by the intraband conductivity as [9]

$$
\sigma=\frac{2 e^{2} k_{B} T}{\left(\tau^{-1}+j \omega\right) \pi \hbar^{2}} \ln \left[2 \cosh \left(\frac{\mu_{c}}{2 k_{B} T}\right)\right]
$$

where $\omega$ is the radian frequency, $\mu_{c}$ is chemical potential, $\tau$ is the electron relaxation time, $T$ is temperature, $e$ is the charge of an electron, $\hbar$ is the reduced Plancks constant, and $k_{B}$ is Boltzmann constant.

The surface impedance $Z_{S}$ of the graphene sheet could be obtained from the surface conductivity $\sigma$ of graphene as $Z_{S}=1 / \sigma=R_{S}+j X_{S}$. By using the equivalent circuit model directly obtained from the analysis of the surface impedance $Z_{S}$, the explanation for the periodic equivalent circuit model of the graphene-based CRLH LWAs is easier.

\section{B. CRLH Leaky-wave Supercell Structures}

Graphene-based CPW technology could be used for achieving the fundamental-mode CRLH LWAs at THz. The parallel-plates are periodic added along their signal line to achieve the series capacitance components $C_{L}$. Their signal line and ground planes are periodically shorted by narrow straight/meander graphene strips to realize the shunt inductance components $L_{L}$.

CPW supercell technology achieves the narrow-band CRLH LWAs in microwave range [8]. It could also provide a method for the perfect satisfication of the closed-stopband condition with some proper optimizations. To achieve the narrow-band closed-stopband CRLH LWAs at THz, graphene-based CPW supercell structures are proposed. By remving the narrow graphene strips from each second section of the conventional

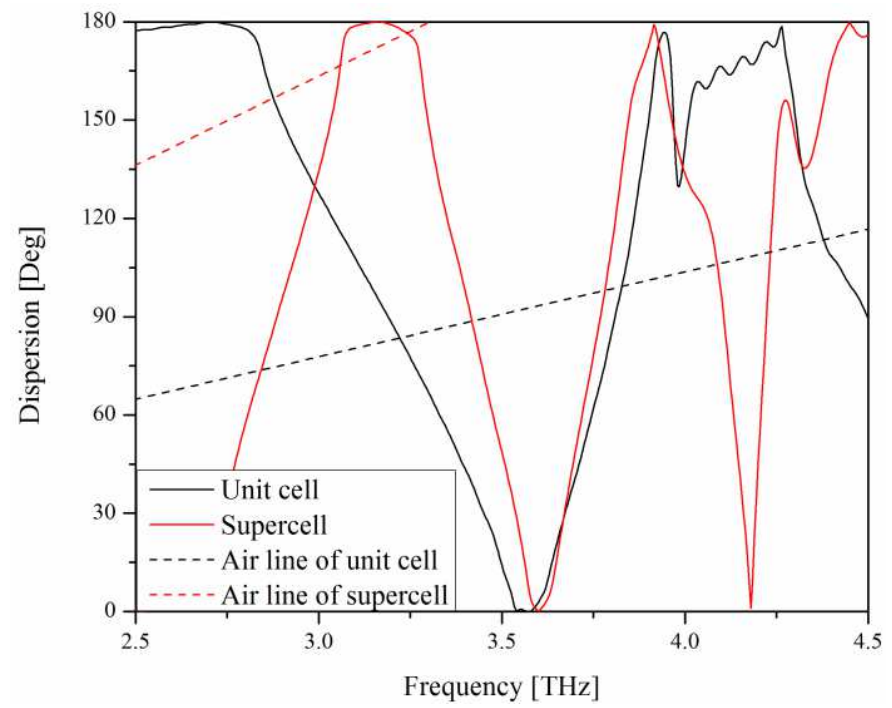

Fig. 2. Dispersion diagram of the designed CRLH leaky-wave structure unit cell and supercell. The optimized parameters of the unit cell are: $g=0.4 \mu \mathrm{m}, G$ $=3.24 \mu \mathrm{m}, W=3.24 \mu \mathrm{m}, L=19.9 \mu \mathrm{m}, s=0.1 \mu \mathrm{m}$; the optimized parameters of the supercell are: $g=0.4 \mu \mathrm{m}, G=3.24 \mu \mathrm{m}, W=3.24 \mu \mathrm{m}, 2 L=36.6 \mu \mathrm{m}, s=0.4$ $\mu \mathrm{m}$.

CRLH leaky-wave unit cell structures, their scanning range is reduced. Moreover, compared with metal material, graphene is more suitable for the $\mathrm{THz}$ antenna applications. Besides, it will provide a strong tunable characteristic to the development of the fundamental-mode CRLH LWAs at THz.

Silica with the dielectric constant $\varepsilon_{r}=3.9$ is chosen as the substrate of these THz LWAs so that the fundamental-mode will be well excited in the CRLH leaky-wave structures rather than surface plasmons.

\section{DiSPERSION ANALYSIS}

The scan angle $\theta$ of the main radiation beam can be expressed by [10]

$$
\theta(\omega)=\sin ^{-1}\left[\frac{\beta(\omega)}{k_{0}}\right]
$$

where $\beta(\omega)$ is the phase constant of this leaky-wave structure, $k_{0}$ is the wavenumber of the free space.

The dispersion diagram for the CRLH leaky-wave structure supercells with a period length $2 L$ can be obtained by the simulated $A B C D$ matrix elements as [8]

$$
\beta \cdot 2 L=\cos ^{-1}\left(\frac{A+D}{2}\right)
$$

Under the detailed illustration above, two graphene-based closed-stopband CRLH unit cell and supercell leaky-wave strucrures with narrow graphene straight strips loaded are designed and printed on the silica with the thickness of $1 \mu \mathrm{m}$. Their chemical potential $\mu_{c}$ and relaxation time $\tau$ of the graphene are $1 \mathrm{eV}$ and $25 \mathrm{ps}$, respectively. CST simulates and exactes their $A B C D$ matrix elements to obtain the dispersion diagram as is shown in Fig. 2.

As Fig. 2 shows, the two designed leaky-wave structures both present a CRLH behavior. Besides, it also proves that the proposed graphene-based $\mathrm{CPW}$ supercell structures have a 


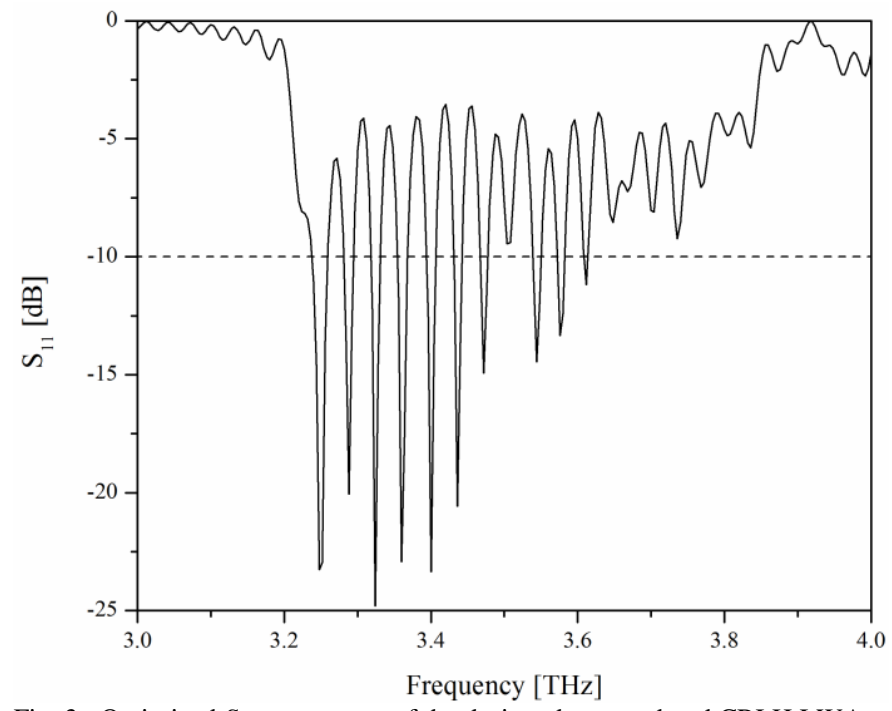

Fig. 3. Optimized $S_{11}$-parameters of the designed narrow-band CRLH LWA.

narrower leaky-mode region compared with the conventional unit cell structures.

The LH and RH region of the graphene-based CRLH supercell structures are an almost seamless transition at around 3.6 THz. It is known that the leaky-mode region is between the intersection of the air line and dispersion curve. Thus, the predicted scanning range of the designed conventional graphene-based CPW unit cell CRLH LWA is over about $-80^{\circ}$ to $90^{\circ}$, and its frequency scanning bandwidth is approximately $0.55 \mathrm{THz}$ from about 3.25 to $3.8 \mathrm{THz}$. But for the designed CPW supercell LWA, its scanning range and frequency bandwidth is narrower. Even more, its actual scanning band is difficult to be predicted because its air line is easy to beyond the range of its dispersion curve.

\section{NARROW-BAND CRLH LWA AT THz}

\section{A. S11-Parameters}

Graphene-based taper line is implemented as the impedance matching technology for this designed narrow-band CRLH LWA with 9 supercells. The final optimized $S_{11}$-parameters of this supercell LWA are shown in Fig. 3.

Fig. 3 shows the $S_{11}$ is below $-10 \mathrm{~dB}$ from about 3.3 to 3.8 THz. Thus the adopted impedance matching network works well enough and most of the energy fed into this structure is radiated to the free space or transformed into the ohmic loss between this band. Moreover, the leaky-mode region of the designed supercell LWA is also between this band. Besides, as Fig. 3 indicates, its $S_{11}$ has a seamless transition at around 3.5 $\mathrm{THz}$ due to the completely satisfaction of the closed-stopband condition which is provided by the employment of the graphene-based CPW supercell technology. Note that, the transition frequency of the designed supercell LWA is at around $3.5 \mathrm{THz}$ as the simulated $S_{11}$-parameters shows.

It is also obsearved that the leaky-mode region and transition frequency obtained from the $S_{11}$-parameters is quite close to the result predicted by the dispersion diagram in Fig. 2. Although there are a slight difference between the predicted results of the designed supercell and its LWA structure, the errors are all in an allowable range.

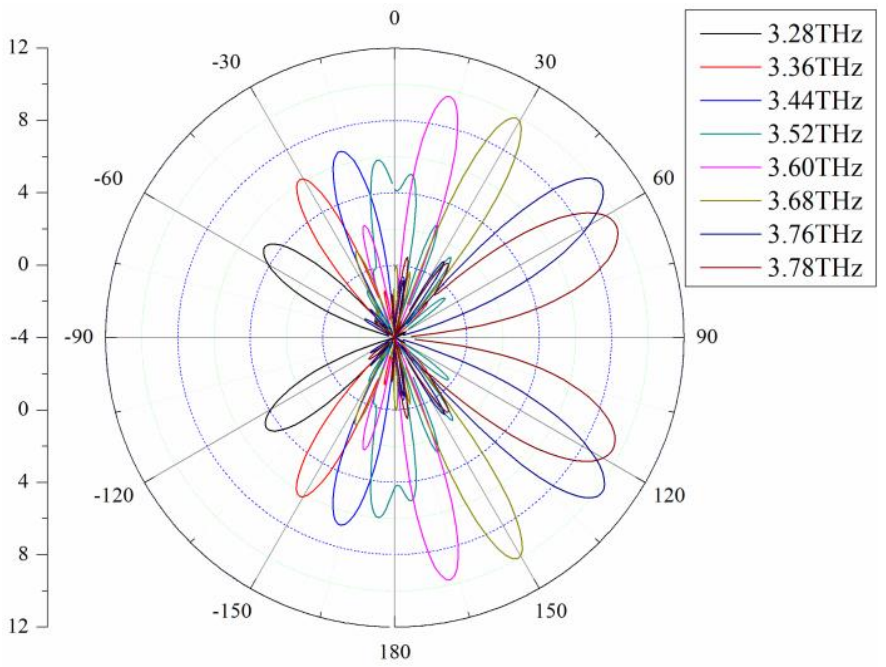

Fig. 4. Radiation pattern of the designed narrow-band CRLH LWA.

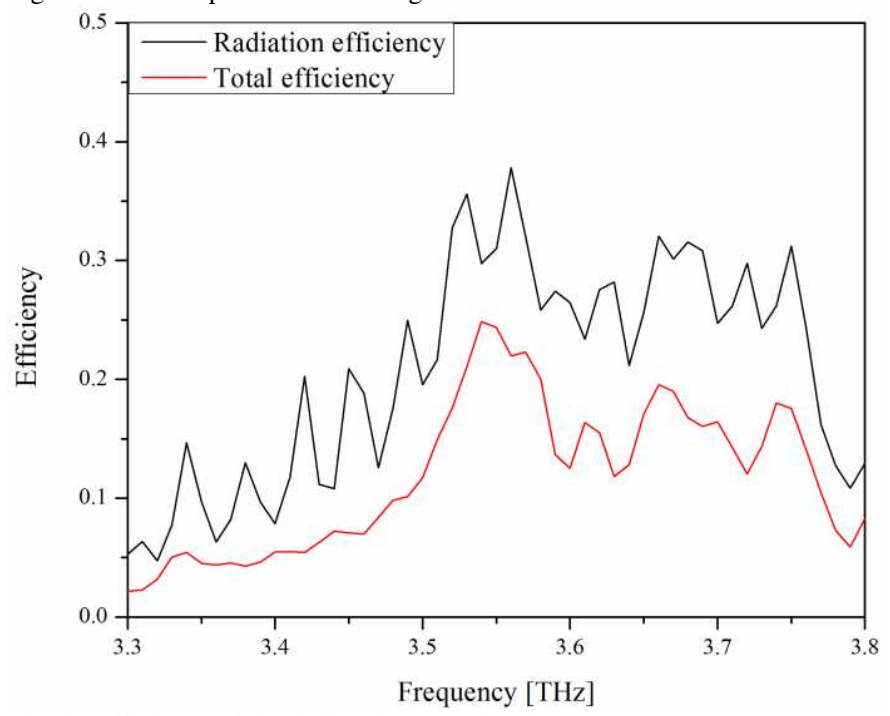

Fig. 5. Efficiency of the designed narrow-band CRLH LWA.

\section{B. Radiation Pattern}

To demonstrate the frequency scanning behavior of the proposed graphene-based CPW supercell CRLH LWAs, CST simulates the farfield radiation performance of the designed CRLH LWA with 9 supercells and the simulation results of the radiation patterns are shown in Fig. 4.

Fig. 4 shows the radiation patterns of the designed LWA from 3.28 to $3.78 \mathrm{THz}$. The angle of its main radiation beam steers as the frequency scanning from about $-58^{\circ}$ to $63^{\circ}$, which is also close to the predicted results from the dispersion diagram. Their radiation beams show that the supercell LWA presents a radiation performance higher than $5 \mathrm{~dB}$ between this band. As Fig. 4 indicates, its transition frequency is at about $3.52 \mathrm{THz}$, which is also close to the prediction from the dispersion diagram. Besides, the magnitude of the radiation beams in the LH region are lower than $8 \mathrm{~dB}$. However, their magnitude in the $\mathrm{RH}$ region is higher than that in the $\mathrm{LH}$ region as Fig. 4 shows. It is worth mentioning that this is also reasonable in most applications.

A seamless transition of the radiation beams is obviously observed at around $3.52 \mathrm{THz}$ because of the more effective 


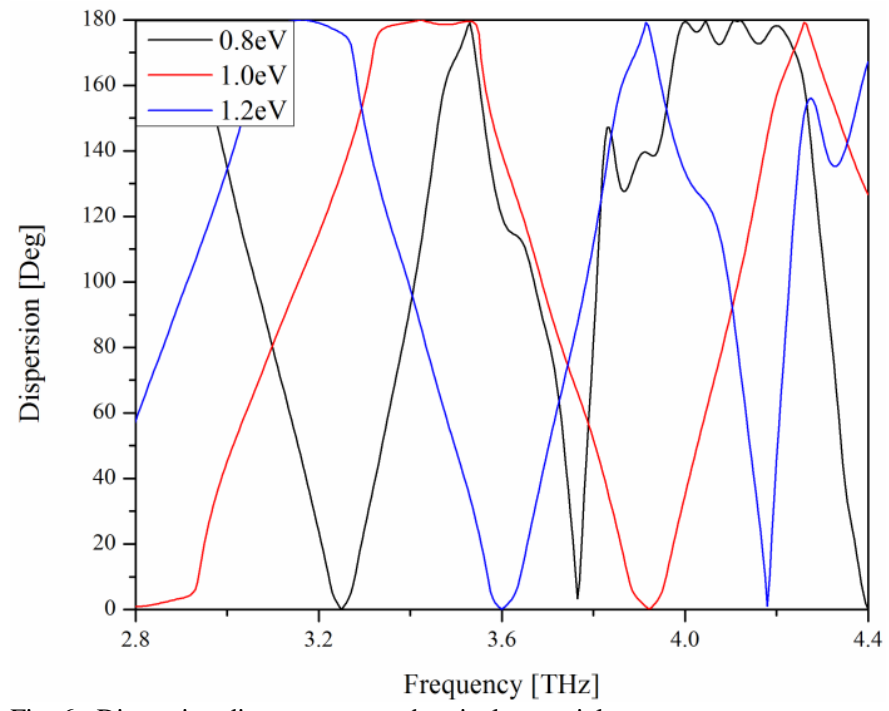

Fig. 6. Dispersion diagram versus chemical potential $\mu_{c}$.

satisfication of the closed-stopband condition compared with the conventional unit cell LWAs.

\section{Efficiency}

After the farfield radiation performance is obtained, the efficiency of the designed supercell LWA is also given as displayed in Fig. 5. Because of the mismatch cause, its radiation efficiency is reasonable higher than the total efficiency.

Again, as Fig. 5 displays, it is obviously observed that the closed-stopband condition is perfectly satisfied without special weak effeciencies appearing.

Moreover, as is reported in Fig. 5, the efficiency is at a lower magnitude than the metal-based LWA in the microwave range because a dispersive model (Drude model) is used to characterize the graphene metamaterial at $\mathrm{THz}$ frequencies. At low $\mathrm{THz}$ band, it is generally adequate to model the material losses with the skin effect [11]. Thus, the efficiencies of the graphene-based LWAs in the THz band will be a reasonable case as the maximum efficiency reaching $40 \%$ at $\tau$ equals 25 ps.

\section{Tunable Characteristics Of Graphene}

\section{A. Dispersion Tuning}

As the illustration for the graphene-based LWAs shows, the transition frequencies of the graphene-based CPW supercell CRLH LWAs are also supposed to be tuned by the chemical potential $\mu_{c}$. In order to understand how a change in the $\mu_{c}$ can affect the dispersion characteristics of a graphene-based CPW supercell CRLH LWA, CST extracts the dispersion relation versus $\mu_{c}$ of the designed CRLH supercell structure and its results is displayed in Fig. 6.

As Fig. 6 shows, with the linear increase of $\mu_{c}$ from 0.8 to 1.2 $\mathrm{eV}$, the transition frequency also increases from about 3.25 to 3.6 and $3.9 \mathrm{THz}$. Therefore, the transition frequency of the proposed supercell CRLH LWAs could also be changed very flexibly according to actual design demand, which is a capability that metal-based CRLH LWAs is absent as well. This characteristic is also expected to provide the proposed CPW supercell CRLH LWAs more application fields than the conventional metal-based CRLH LWAs.

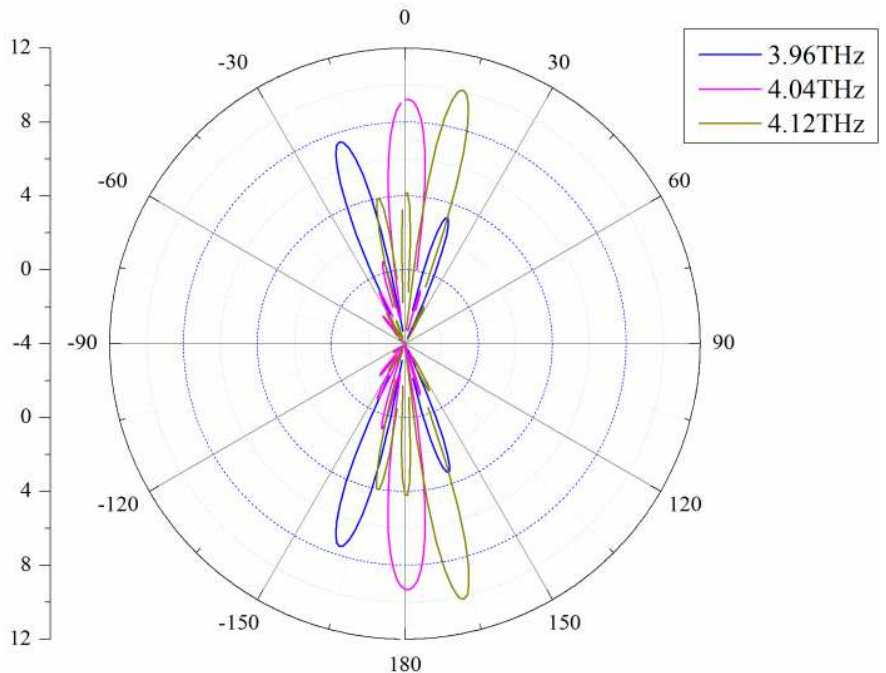

(a)

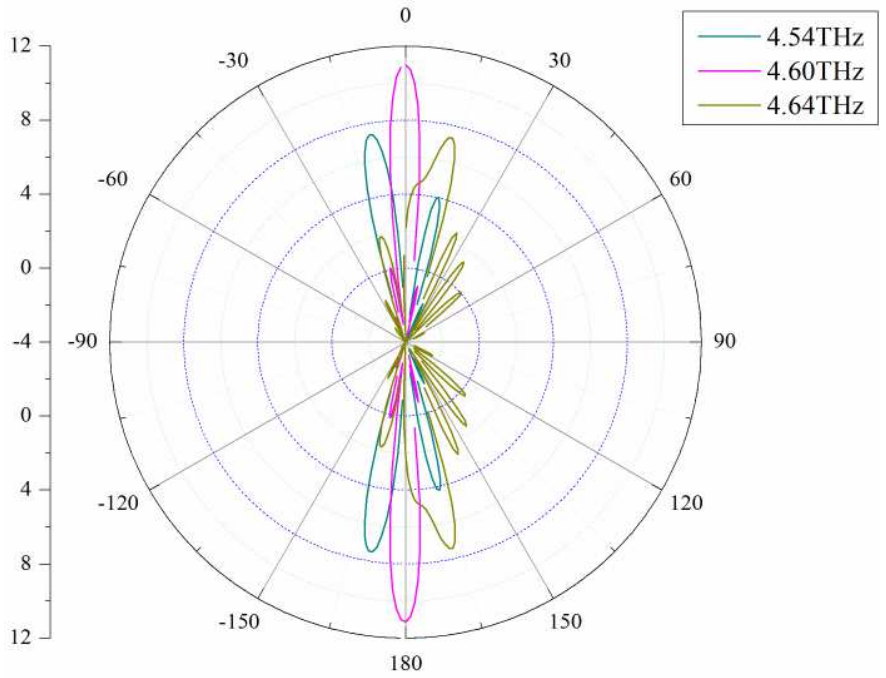

(b)

Fig. 7. Radiation patterns tuning through chemical potential as (a) $\mu_{c}=1.5 \mathrm{eV}$, (b) $u_{r}=2 \mathrm{eV}$.

\section{B. Radiation Patterns Tuning}

Fig. 7 confirms that the radiation patterns of the proposed supercell LWAs could be tuned by different chemical potentials $\mu_{c}$ as 1.5 and $2 \mathrm{eV}$, respectively. Consider Figs. 4 and 7 , it could be obviously observed that the transition frequency of the designed supercell CRLH LWA is shifted from about 3.52 to 4.28 and $4.84 \mathrm{THz}$ by tuning $\mu_{c}$.

Note that, due to the employment of graphene, the main radiation beams of the proposed supercell LWAs are supposed to be changed through $\mu_{c}$ varying as the LWAs in [11], [13]. To describe how this change occurs, the operating frequency of the designed graphene-based CPW supercell LWA is fixed at 3.52 $\mathrm{THz}$ which can be extended to the cases of other operating frequencies. The simulated radiation patterns gives the scanning angle of its main radiation beams versus $\mu_{c}$ as shown in Fig. 8.

As Fig. 8 shows, the main radiation beam of the designed supercell CRLH LWA steers obviously as $\mu_{c}$ varying from 0.9 to $1.1 \mathrm{eV}$. As is observed from the simulated results, the proposed scheme provides the graphene-based CPW supercell CRLH LWAs the scanning capability as chemical potential $\mu_{c}$ varying at $\mathrm{THz}$ by employing graphene as well. 


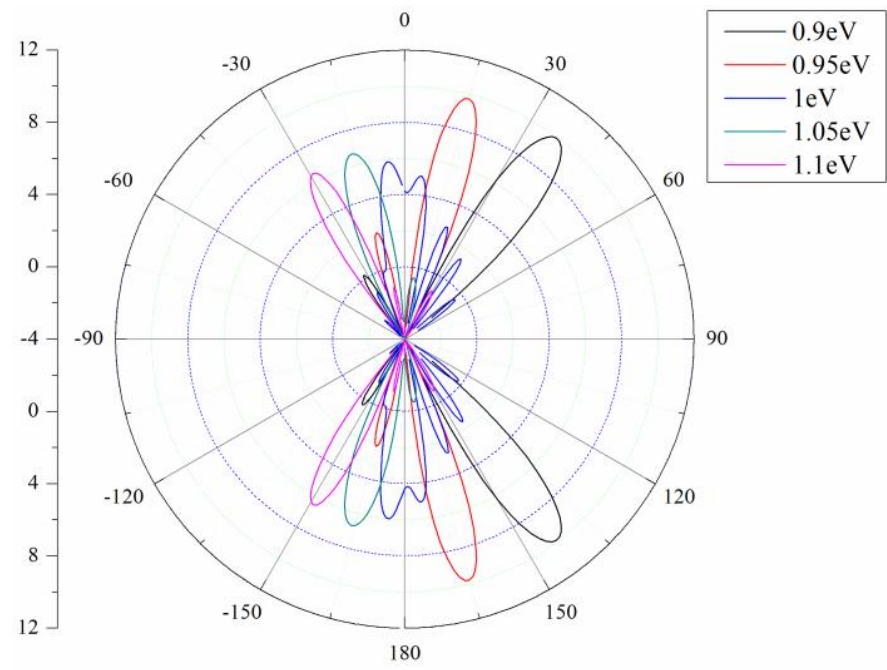

Fig. 8. Radiation patterns versus chemical potential $\mu_{c}$ at $3.52 \mathrm{THz}$.

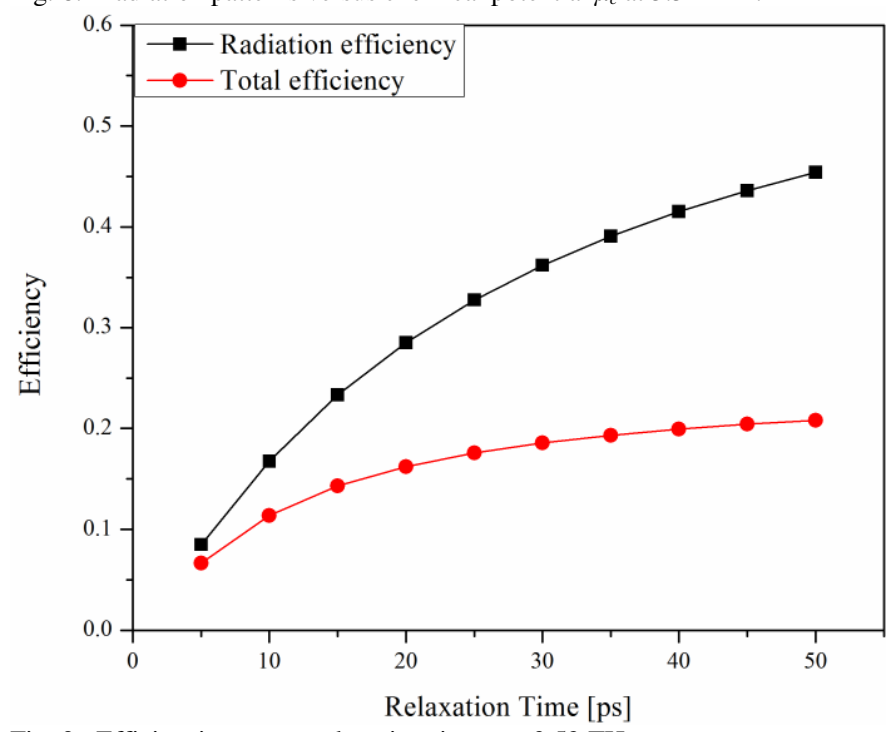

Fig. 9. Efficiencies versus relaxation time $\tau$ at $3.52 \mathrm{THz}$.

\section{Efficiency Tuning}

Again, as well as the illustration for the graphene-based LWAs shows, the efficiency of the proposed CPW supercell LWA is also supposed to be tuned by $\tau$.

To understand the change of the efficiency versus $\tau$ of the proposed supercell CRLH LWAs, we also fix the operating frequency of the designed graphene-based CPW supercell CRLH LWA at $3.52 \mathrm{THz}$. Its simulated results of the radiation and total efficiency are displayed in Fig. 9.

As is shown in Fig. 9, the efficiency increases obviously as the relaxation time $\tau$ increasing. However, the efficiency will reach a limitation above $50 \%$. Note that, we could choose a suitable $\tau$ to obtain the efficiency that satisfies the actual design demand. Again, the maximum radiation efficiency is higher than the presented efficiency of $46 \%$ in [11] with a high relaxation time.

\section{Conclusion}

A simple scheme for realizing the $\mathrm{THz}$ graphene-based closed-stopband CRLH LWAs is proposed in this paper. The narrow-band CRLH leaky-wave structures is achieved by remving the narrow graphene strips from each second section of the graphene-based CRLH leaky-wave unit cell structures. To demonstrate the proposed structures, a CRLH leaky-wave supercell structure is designed and its dispersion relation leaky-mode regions is extracted. The simulation results indicate that the scanning angles of its main radiation beams also steers obviously with the frequencies varying and a backfire-to-endfire radiation capability is achieved at $\mathrm{THz}$. Besides, the proposed supercell CRLH LWAs could achieve the closed-stopband condition more effective at the broadside angle. Furthermore, the strong tunable characteristics of the proposed supercell LWAs are discovered at THz as well. Hence, the proposed supercell LWAs would futher improve the performance of beam-steering antennas at $\mathrm{THz}$.

\section{Funding Information}

This work was supported by the National Natural Science Foundation of China (61475084) and China Natural Science Foundation of Shandong Province (ZR2017MA039).

\section{Authors' Contributions}

Data curation, writing original draft, writing review and editing: [Muzhi Gao]; data and results discussion: [Kang Li, Fanmin Kong, Gaoyang Zhu, Huawei Zhuang].

\section{Compliance with Ethical Standards}

Competing Interests The authors declare that they have no confict of interest.

Consent to Participate The author gives his consent to participate.

Consent to Publish The author gives his consent to publish.

\section{Data Availability}

The data that support the findings of this study are available from the corresponding author upon reasonable request.

\section{REFERENCES}

[1] Kemp MC et al (2003) Security applications of terahertz technology. Proc SPIE 5070(44): 44-52

[2] Novoselov KS, Fal V, Colombo L, Gellert P, Schwab M, Kim K (2012) A roadmap for graphene. Nature 490(7419): 192-200

[3] Hon PWC, Tavallaee AA, Chen QS, Williams BS, Itoh T (2012) Radiation Model for Terahertz Transmission-Line Metamaterial Quantum-Cascade Lasers. IEEE Trans THz Sci Technol 2(3): 323-332

[4] Hon PWC, Liu Z, Itoh T, Williams BS (2013) Leaky and bound modes in terahertz metasurfaces made of transmission-line metamaterials. Appl Phys Lett 113(3): 033105

[5] Liu Z, Hon PWC, Tavallaee AA, Itoh T (2012) Terahertz composite right-left handed transmission-line metamaterial waveguides. Appl Phys Lett 100(7): 071101

[6] Tavallaee AA, Hon PWC, Chen QS, Itoh T, Williams BS (2013) Active terahertz quantum-cascade composite right/left-handed metamaterial. Appl Phys Lett 102(2): 021103

[7] Chu DA, Hon PWC, Itoh T, Williams BS (2016) Feasibility of graphene CRLH metamaterial waveguides and leaky wave antennas. J Appl Phys 120(1): 013103

[8] Mehdipour A, Eleftheriades GV (2014) Leaky-wave Antennas Using Negative-Refractive-Index Transmission-Line Metamaterial Supercells. IEEE Trans Antennas Propag 62(8): 3929-3942

[9] Fuscaldo W, Burghignoli P, Baccarelli P, Galli A (2017) Graphene Fabry-Perot Cavity Leaky-Wave Antennas: Plasmonic Versus Nonplasmonic Solutions. IEEE Trans Antennas Propag 65(4): 1651-1660 
[10] Caloz C, Itoh T (2005) Electromagnetic Metamaterials: Transmission Line Theory and Microwave Applications. John Wiley, New York

[11] Memarian M, Eleftheriades GV (2015) Dirac leaky-wave antennas for continuous beam scanning from photonic crystals. Nature Communications 6: 5855

[12] Esquius-Morote M, Gómez-D'́az J, Perruisseau-Carrier J (2014) Sinusoidally Modulated Graphene Leaky-Wave Antenna for Electronic Beamscanning at THz. IEEE Trans THz Sci Technol 4(1): 116-122

[13] Wang XC, Zhao WS, Hu J, Yin WY (2015) Reconfigurable Terahertz Leaky-Wave Antenna Using Graphene-Based High-Impedance Surface IEEE Trans Nanotechnol 14(1): 62-69 
Figures
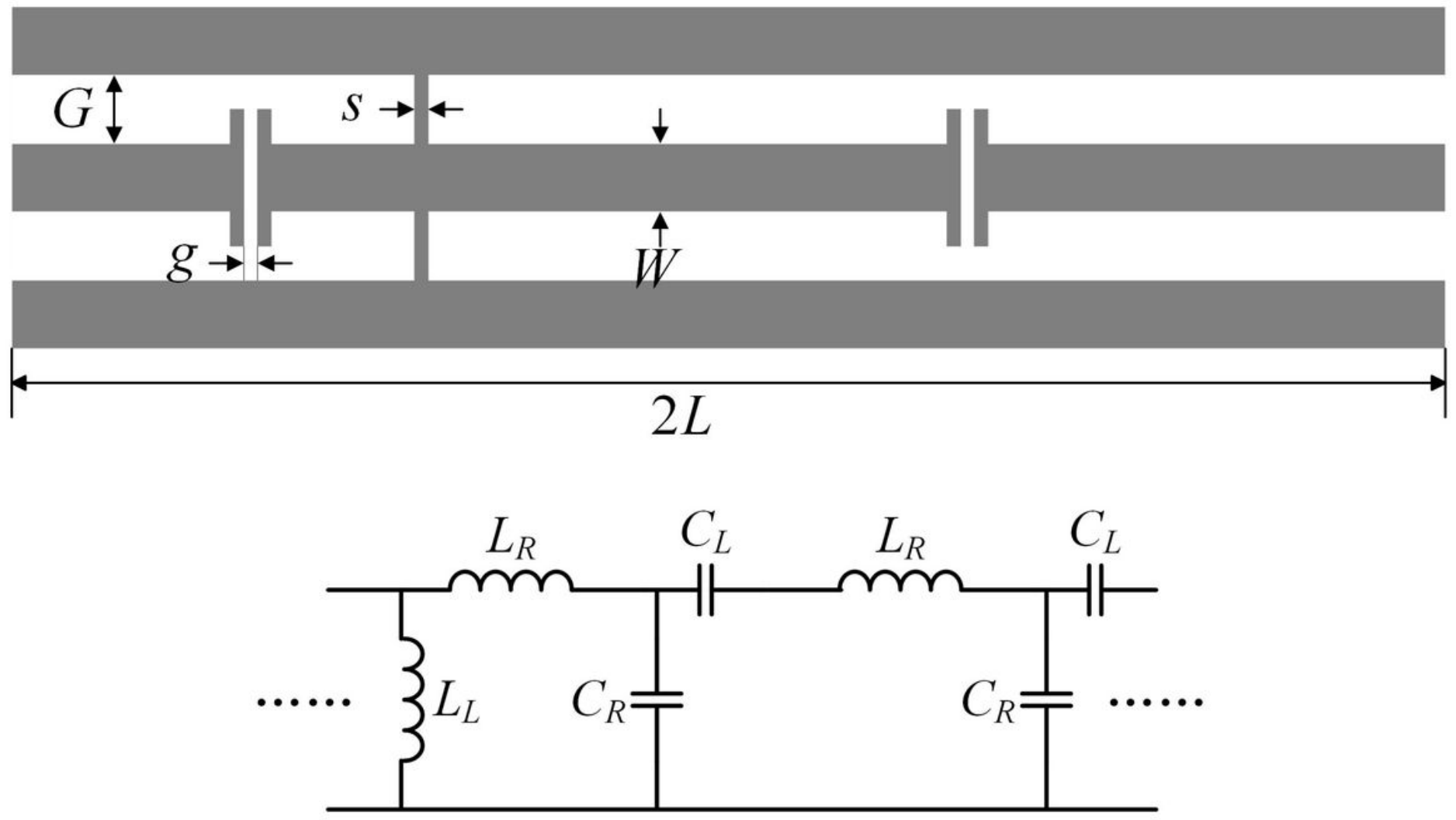

Figure 1

The proposed supercell CRLH leaky-wave structures. (a) Layout. (b) Equivalent circuit model. 


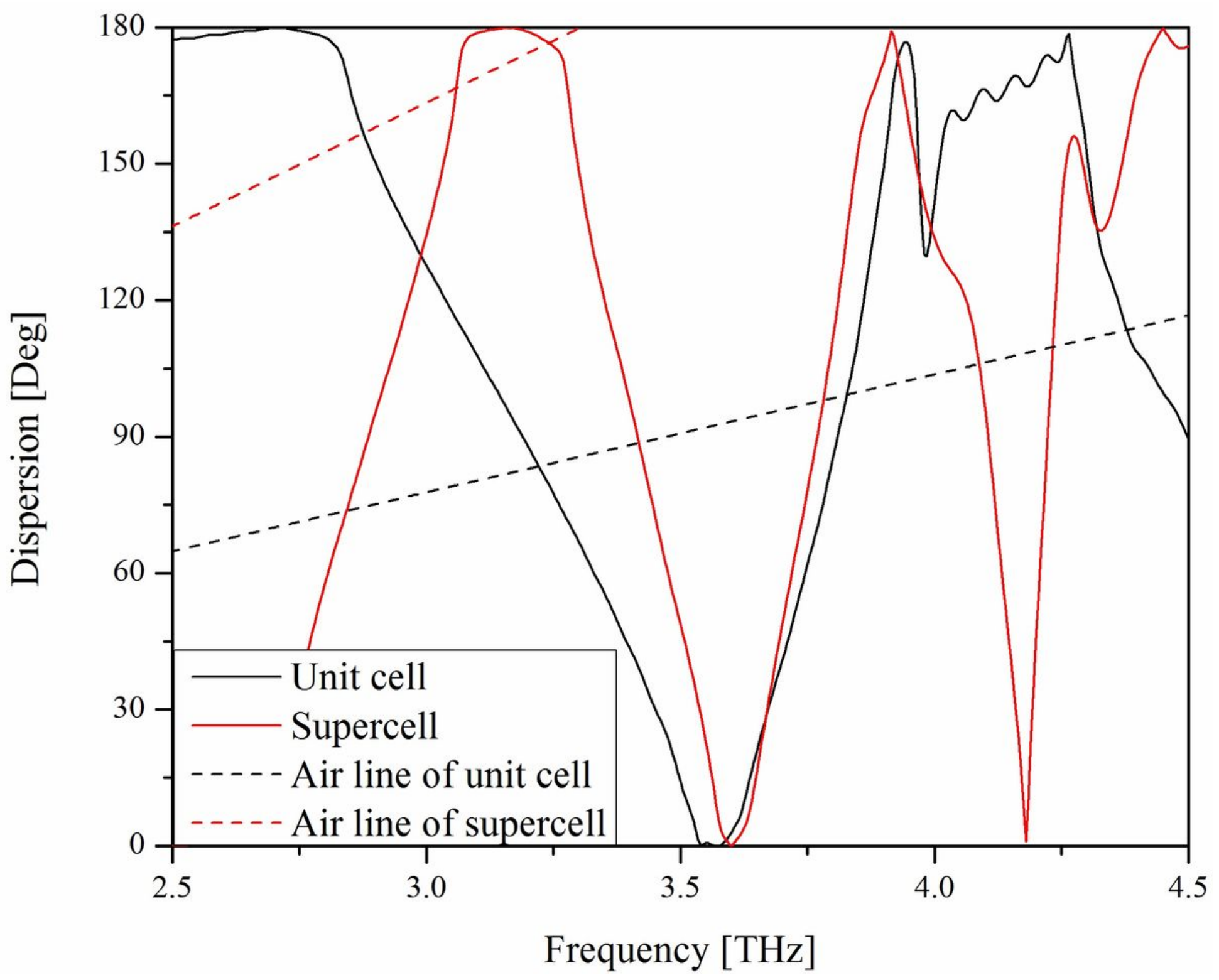

Figure 2

Dispersion diagram of the designed CRLH leaky-wave structure unit cell and supercell. The optimized parameters of the unit cell are: $\mathrm{g}=0.4 \mu \mathrm{m}, \mathrm{G}=3.24 \mu \mathrm{m}, \mathrm{W}=3.24 \mu \mathrm{m}, \mathrm{L}=19.9 \mu \mathrm{m}, \mathrm{s}=0.1 \mu \mathrm{m}$; the optimized parameters of the supercell are: $\mathrm{g}=0.4 \mu \mathrm{m}, \mathrm{G}=3.24 \mu \mathrm{m}, \mathrm{W}=3.24 \mu \mathrm{m}, 2 \mathrm{~L}=36.6 \mu \mathrm{m}, \mathrm{s}=0.4$ $\mu \mathrm{m}$. 


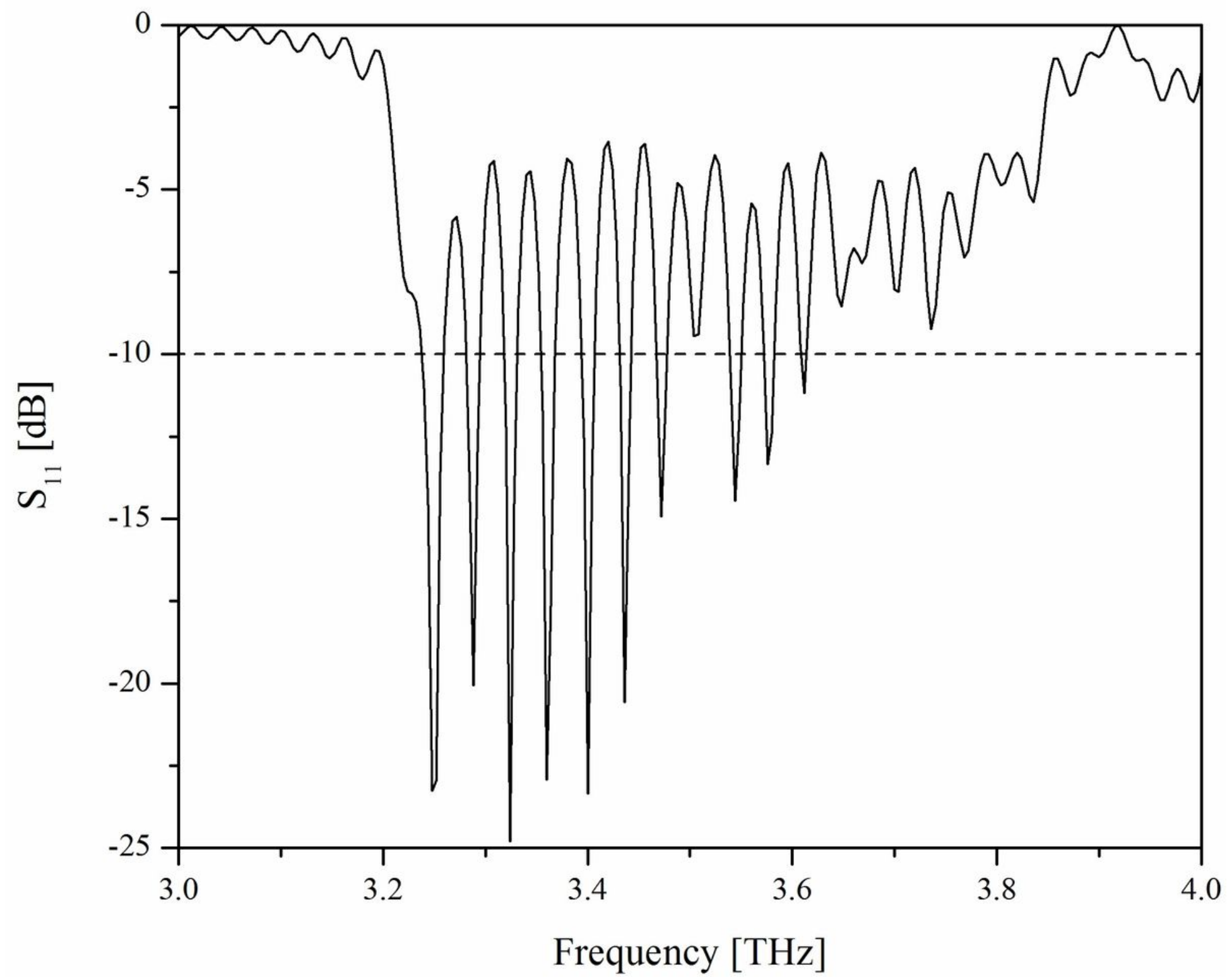

Figure 3

Optimized S11-parameters of the designed narrow-band CRLH LWA. 


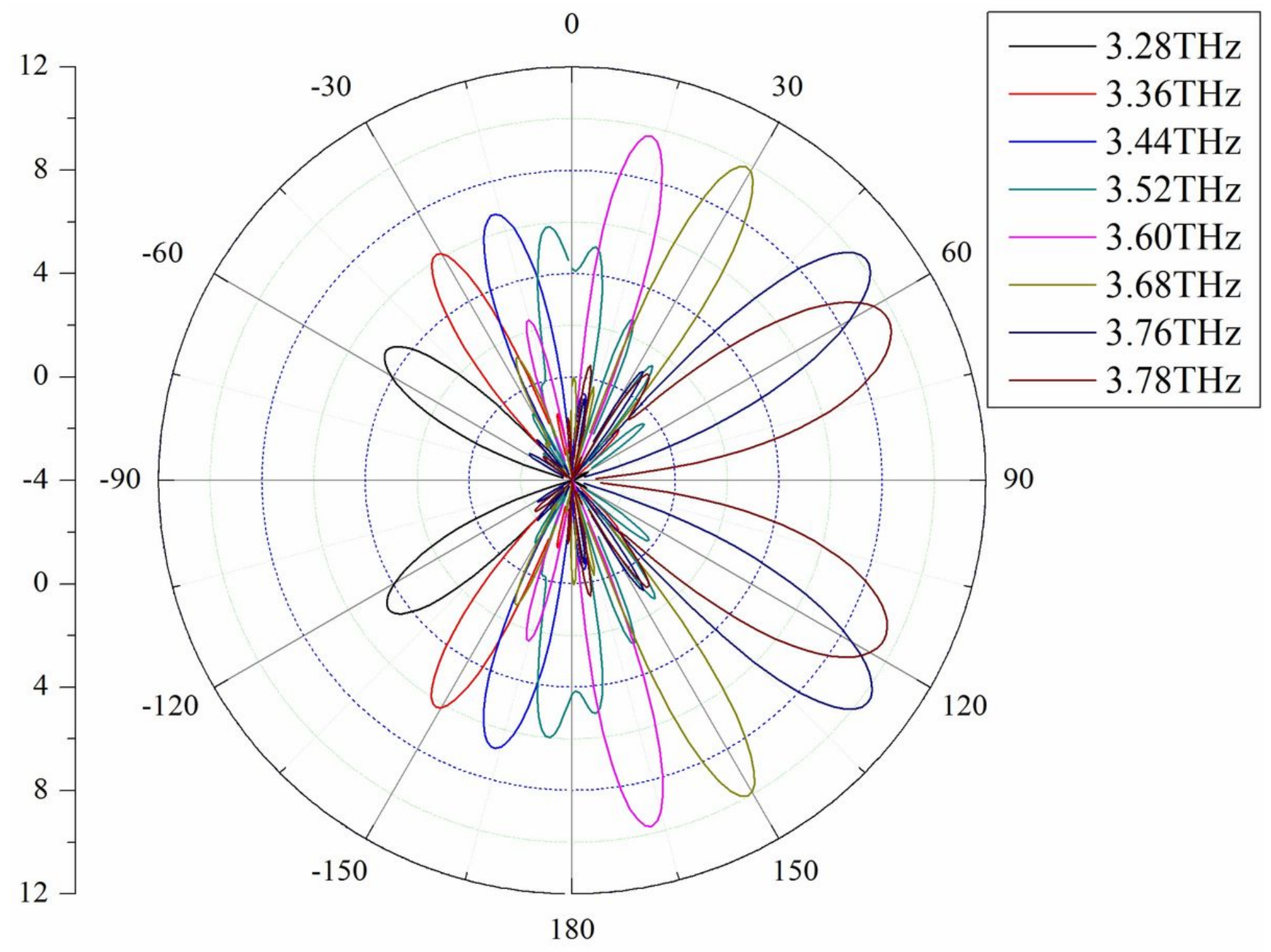

Figure 4

Radiation pattern of the designed narrow-band CRLH LWA. 


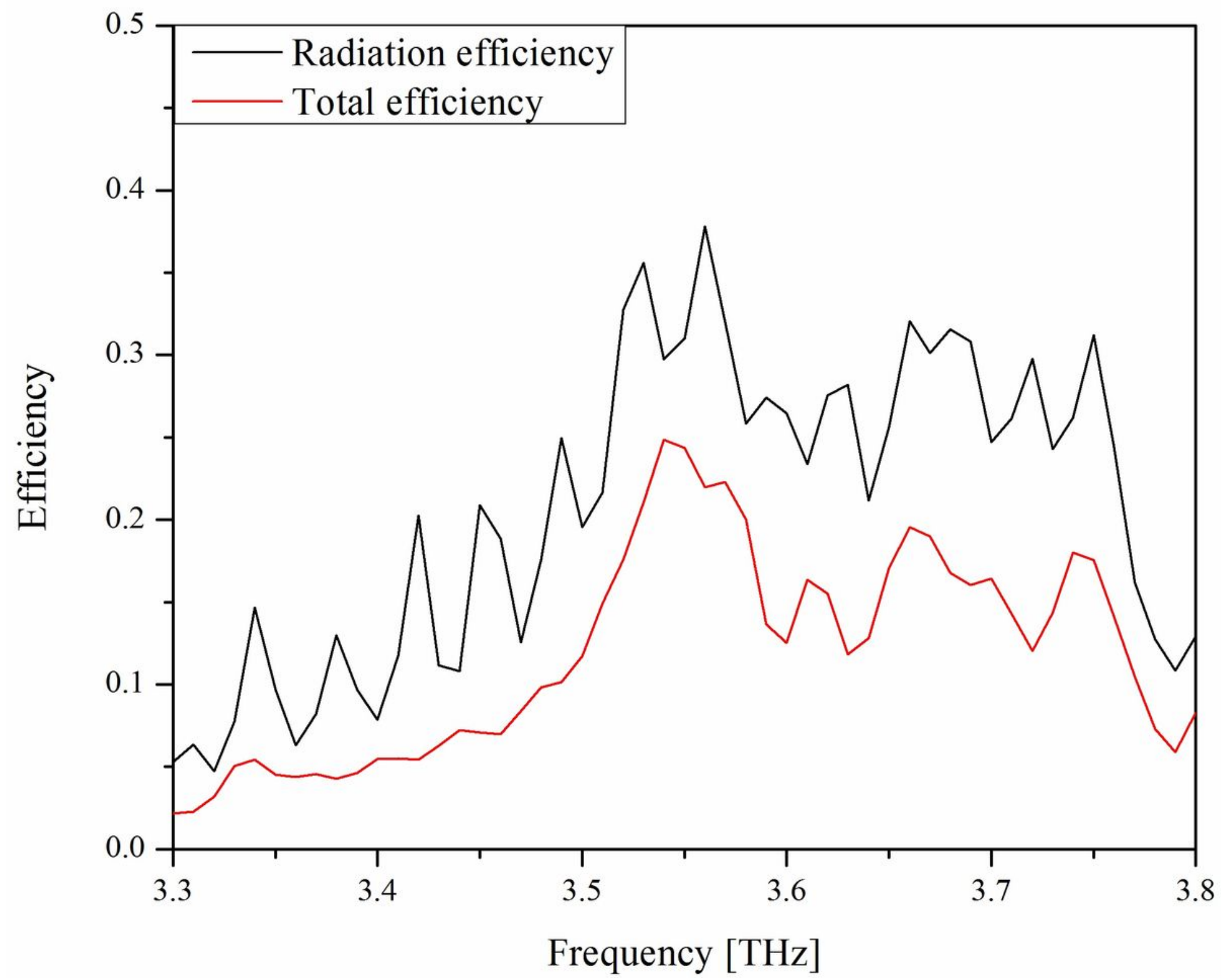

Figure 5

Efficiency of the designed narrow-band CRLH LWA. 


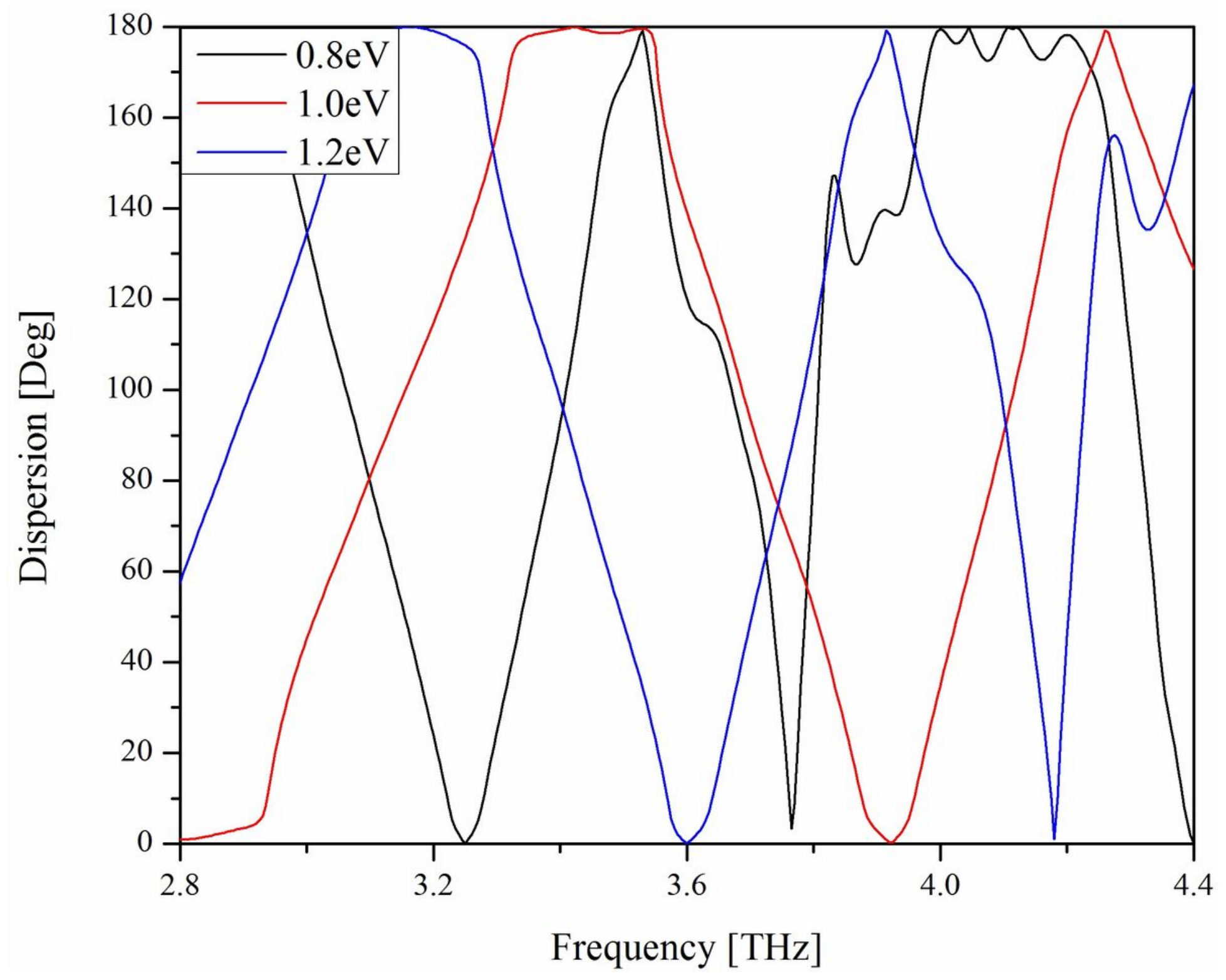

Figure 6

Dispersion diagram versus chemical potential $\mu \mathrm{c}$. 

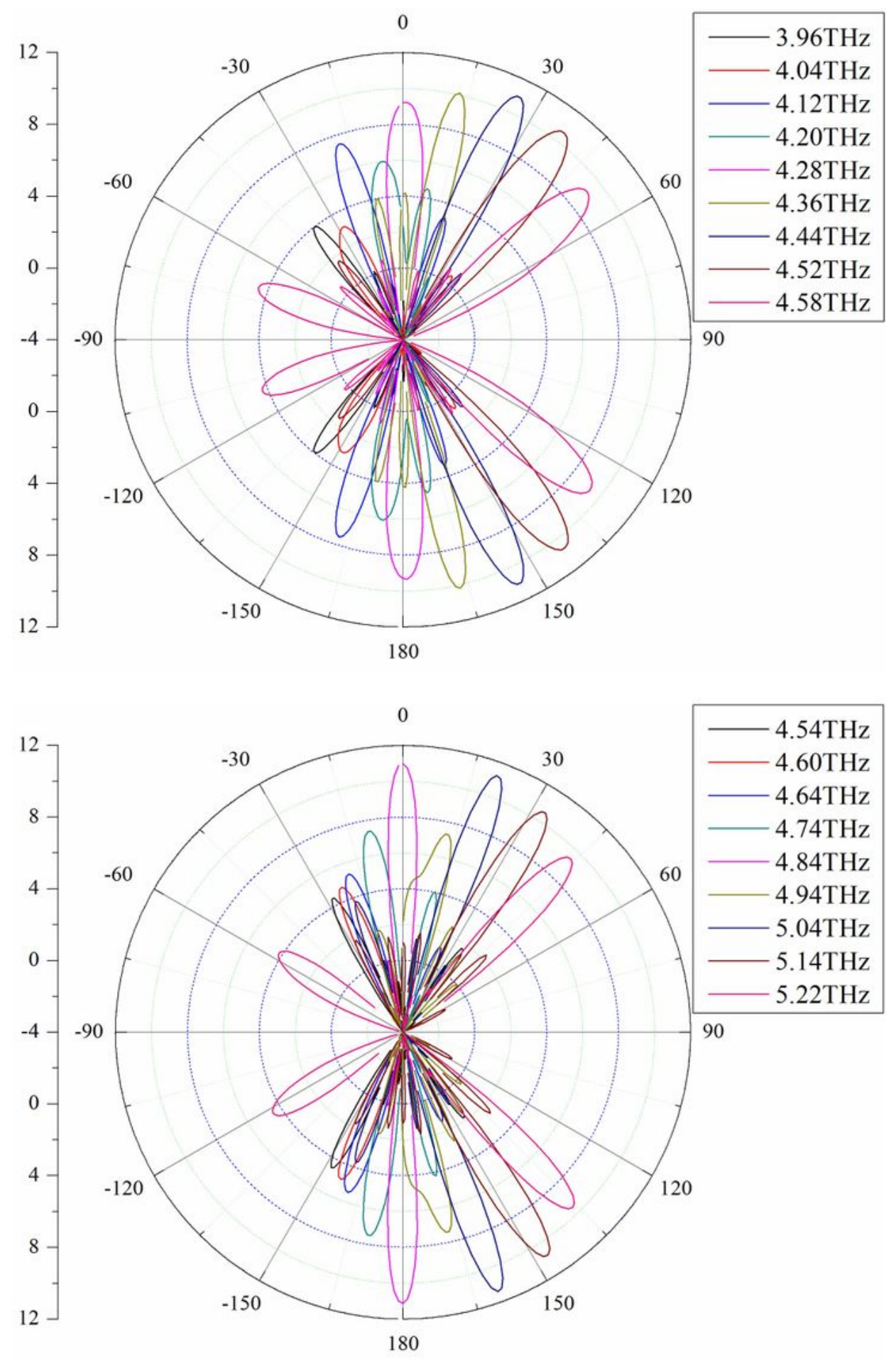

\section{Figure 7}

Radiation patterns tuning through chemical potential as (a) $\mu \mathrm{c}=1.5 \mathrm{eV},(\mathrm{b}) \mu \mathrm{c}=2 \mathrm{eV}$. 


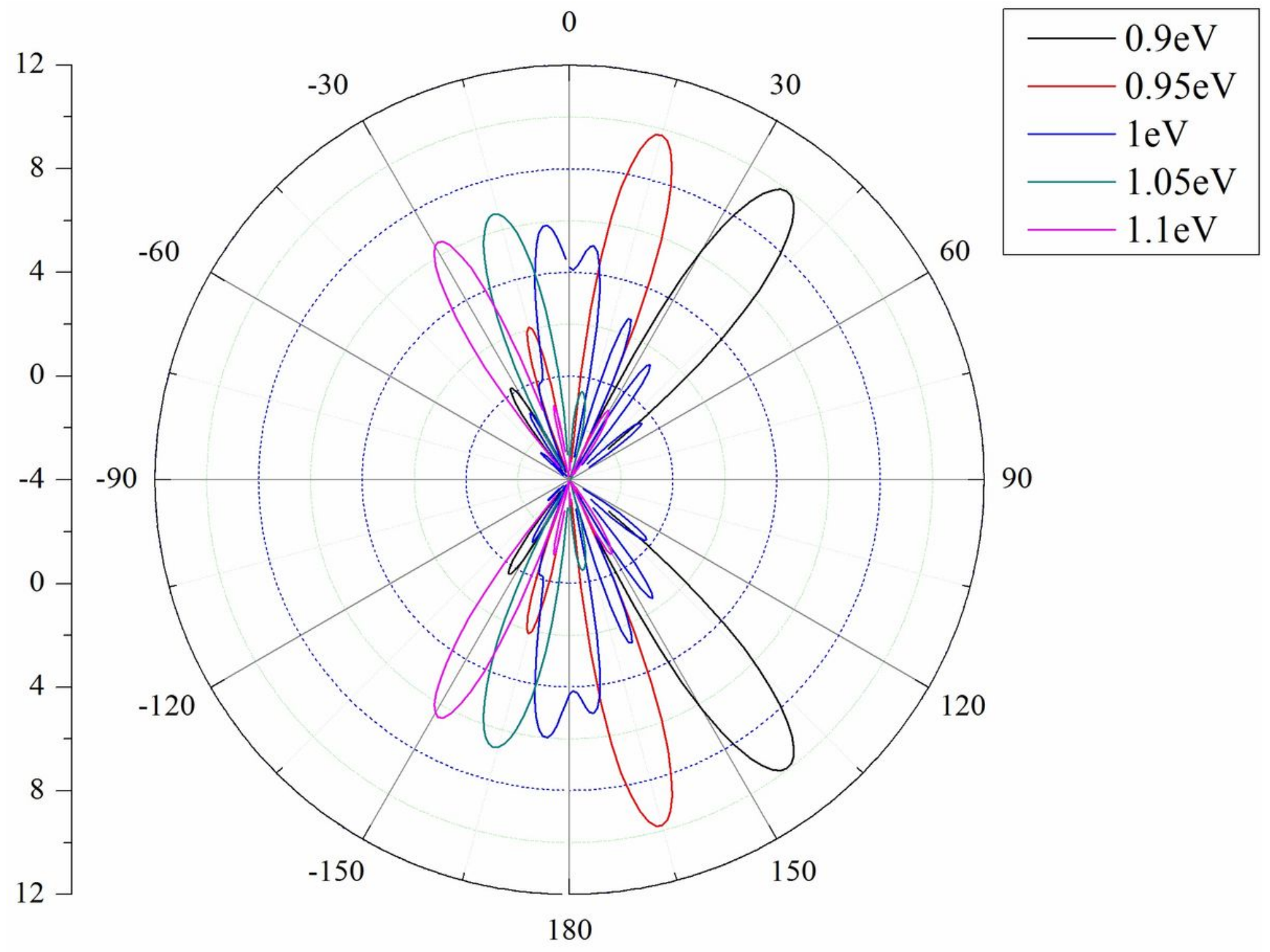

Figure 8

Radiation patterns versus chemical potential $\mu \mathrm{c}$ at $3.52 \mathrm{THz}$. 


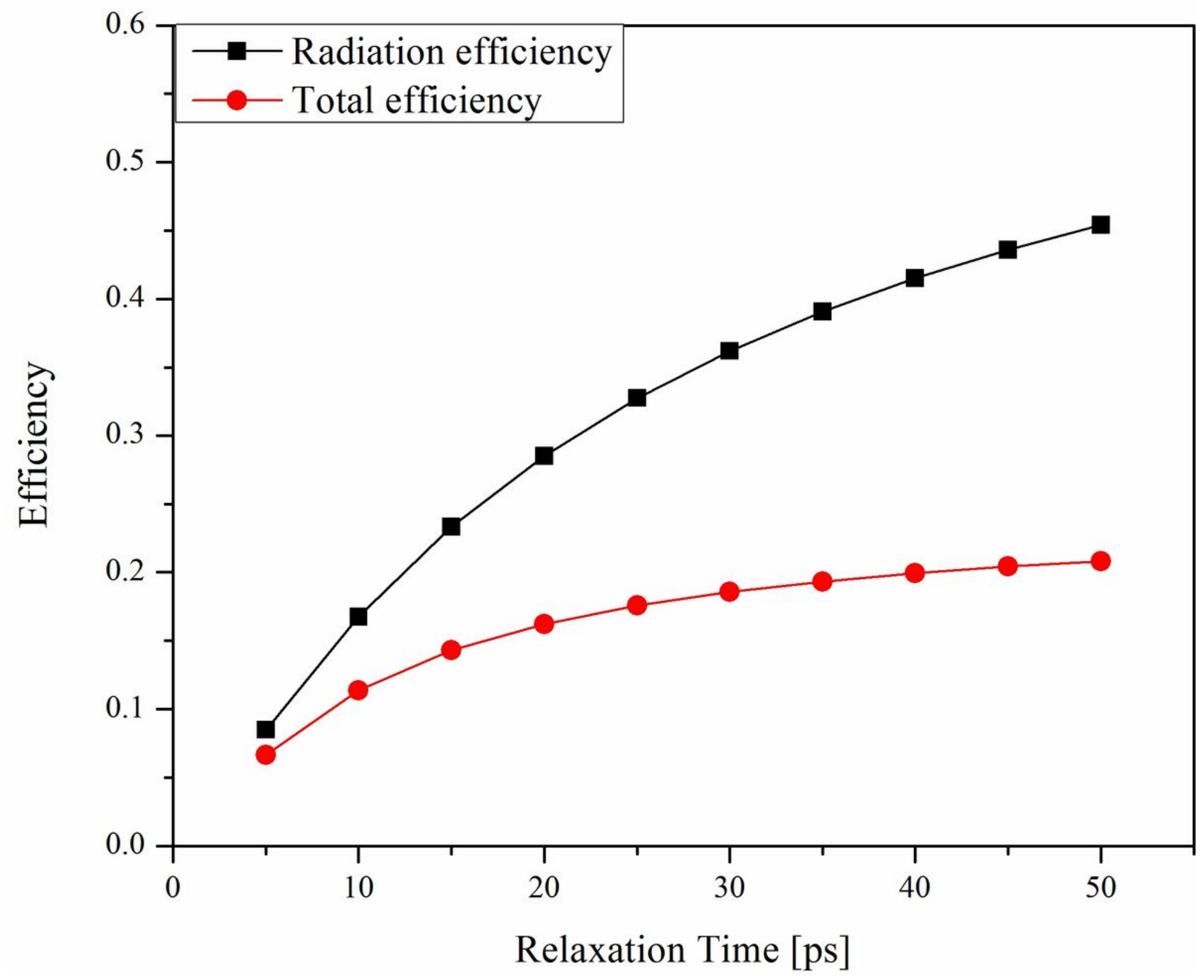

Figure 9

Efficiencies versus relaxation time $\tau$ at $3.52 \mathrm{THz}$. 